\title{
APLICACIONES DE LA TEORÍA DE JUEGOS EN EL PROCESO DE DIRECCIÓN Y ADMINISTRACIÓN ESTRATÉGICA DE EMPRESAS
}

\author{
APPLICATIONS OF GAME THEORY IN THE PROCESS OF STRATEGIC \\ MANAGEMENT AND ADMINISTRATION OF COMPANIES
}

MARÍA AUXILIADORA VARGAS VALDIVIEZO

Universidad Católica de Santiago de Guayaquil. maria.vargas06@cu.ucsg.edu.ec

RESUMEN

El presente trabajo tiene como propósito determinar las Aplicaciones de la Teoría de Juego en el Proceso de Dirección y Administración Estratégica de Empresas, por ello se realizó una revisión de la literatura de las aportaciones conceptuales, perspectivas, hallazgos y contribuciones a la práctica administrativa que han realizado varios autores citados de forma lógica y ordenada en el presente documento, que para lograr el propósito planteado ha centrado su atención en revisar las aplicaciones en lo referente a las Finanzas, Marketing, Negociación y Administración de Operaciones. Y se establece como conclusión principal que la Teoría de Juegos tiene relación con la práctica administrativa debido a que brinda objetividad en cada uno de los procesos de la función administrativa; esto es, en lo referente a la toma de decisiones. De la misma forma muestra que existe una limitación que no se puede dejar de mencionar y es que al ser una teoría que fundamenta su estructura en modelos matemáticos, los cuales son visiblemente rígidos en lo que se refiere a las actividades humanas en las que se involucra comportamiento, lo que hace que las sugerencias o consejos proporcionados por la aplicacion de esta teoría no alcancen niveles altos de certeza.

PALABRAS CLAVE: teoría de juegos, estrategia, administración estratégica, proceso de administración estratégica.

\begin{abstract}
The purpose of this study is to determine the applications of Game Theory in Process Management and Strategic Business Administration, specifically this work has focused on reviewing applications regarding Finance, Marketing, Negotiation and Administration Operations. For which a literature review of the conceptual contributions, perspectives, findings and contributions to the administrative practice which has made several authors cited and related in an orderly and logical herein was performed. The main conclusion reached in this paper is that game theory is related to the administrative practice because it provides objectivity in each of the processes of administrative function, ie, in decision-making. However, also it shows that there is a limitation that can not fail to mention is that when a theory that bases its structure on mathematical models, these are obviously not adaptable when it comes to human activities in which behavior is involved, making suggestions or advice provided by the application of this theory to lose not reach strong levels of certainty.
\end{abstract}

KEYWORDS: game theory, strategy, strategic management, strategic management process. 
INTRODUCCIÓN

En este estudio se analizan experiencias anteriores que brindan información de la Aplicación de la Teoría de Juegos en el Proceso de Dirección y Administración Estratégica de Empresas, realizando una revisión de trabajos que nos describen ¿Qué es la Teoría de Juegos?, ¿Cuál es el alcance de la Teoría de Juegos?, ¿Cuál es su aplicación en el ámbito organizacional?, ¿Cómo se relaciona la Teoría de Juegos con la estrategia?, y ¿Cómo se aplica la Teoría de Juegos en el proceso de administración estratégica?. Todas preguntas en algún momento se han realizado en el ámbito administrativo tanto desde la perspectiva de los administradores, de los académicos que han investigado y brindado su trabajo como contribución en el avance del conocimiento administrativo.

Es muy importante que a través de un análisis detallado de la literatura conocer acerca de la aplicación de la Teoría de Juegos en el proceso Administrativo, como éste se ha ido adaptando a los avances de la sociedad y a las exigencias de la globalización y ha evolucionado para ofrecer nuevas perspectivas que permiten a los administradores tener bases para la toma de decisiones de forma estratégica y sobretodo fundamentada. Este tema tiene relevancia debido al dinamismo del mundo empresarial en el cuál se requiere de un proceso administrativo lo suficientemente estructurado que siga un plan y que además sea estratégico para alcanzar los objetivos propuestos por la organización, aislando la subjetividad en la toma de decisiones.

\section{MARCO TEÓRICO Y CONCEPTUAL}

La teoría de juegos es parte de las matemáticas aplicadas en la cual se emplea modelos para resolver los problemas planteados, esta teoría tiene sus orígenes en el área militar y es utilizada en varios ámbitos de la ciencia. La teoría matemática de juegos fue inventada por Von Neumann \& Morgenstern, (1944) y posteriormente el científico Nash (1950) realizó sus aportes. Una definición completa de teoría de juegos es aquella en la que "para que un juego o situación estratégica esté definido completamente, deben establecerse: (a) jugadores, (b) reglas y estructura temporal del juego, (c) estructura de información del juego, (d) resultados del juego y (e) juegos (Tarziján \& Paredes, 2006, p. 197). Esta teoría fue desarrollada para ayudar a los administradores a comprender ciertas situaciones que se presentan debido a que "se argumenta que existe un potencial significa- tivo para el desarrollo de modelos metafóricos que capturan las amplias características cualitativas de los ajustes que implican interacciones estratégicas"(Saloner, 1991).

Que las empresas son racionales y que los administradores toman decisiones empresariales en las que se evidencia razonamiento y pensamiento estratégico son supuestos que subyacen de esta teoría, de donde se puede definir que "el negocio es un juego de altas apuestas" (Brandenburger \& Nalebuff, 1995, p. 57). Algunas de las expresiones en los negocios no son propias sino adoptadas desde la milicia y de los deportes; no obstante el fin principal de los administradores en las empresas es ganar, sin que ello signifique que las otras empresas van a perder. Sin embargo, los gerentes deben realizar jugadas (desarrollar estrategias) que coadyuven a lograr los objetivos trazados, porque "la esencia del éxito radica en asegurarse de que está jugando el juego correcto. ¿Cómo sabes si es el juego correcto? Para ello los autores han desarrollado un marco que se basa en las ideas de la teoría de juegos" (Brandenburger \& Nalebuff, 1995, p. 57).

La identificación de los cinco elementos que determinan un juego permite a los administradores definir "la interdependencia estratégica entre las empresas puede darse en forma simultánea o secuencial, y hay ocasiones en las que se dan ambos tipos, las interacciones simultáneas es cuando las empresas pueden tomar decisiones al mismo tiempo" (Tarziján \& Paredes, 2006, p. 189). También hay interdependencia estratégica secuencial las cuáles se refieren a que los intervinientes desconocen información que hace posible una decisión (Tarziján \& Paredes, 2006). Esto parece indicar que el administrador puede variar el juego (la estrategia) lo que a la vez generará que el resultado pueda variar (Brandenburger \& Nalebuff, 1995).

Por otra parte también hubo quienes creyeron que la aplicación de la teoría de juegos no era adecuada para el sector empresarial, debido a que "la teoría de juegos es difícil de usar y de probar, amenaza con no explicar nada, genera modelos personalizados de ajustes locales en lugar de regularidades generales, y ofrece limitados consejos a los administradores (Camerer, 1991). Estas críticas se derivan de creer que la teoría de juegos utiliza poca racionalidad en el cálculo del equilibrio (Camerer, 1991). No obstante, las empresas asumen decisiones o estrategias de actuación que generarán un resultado en el juego, ahora no todos los resulta- 
dos producen equilibrio y esto se debe a que las decisiones dependen del contexto en el que se las tome (Ansoff, 1991). En referencia a su aplicabilidad en el sector empresarial se necesita de cierta confiabilidad por lo que se denominó Teoría de Juegos Corporativos debido a que tiene cierta formalidad que permite exigibilidad ante determinada acción y hace posible negociación $\mathrm{y}$ en este aspecto Nash introduce un valioso aporte que viabiliza una negociación justa entre los involucrados en el juego (Lippman \& Rumelt, 2003)

Las definiciones de las amenazas, fortalezas, debilidades y oportunidades de la empresa con el entorno (Andrews, 1971; Ansoff, 1991) estos aportes contribuyeron a la definición de "Una estrategia es un conjunto de políticas" (Tilles, 1963) a partir de esta definición se plantea el concepto dinámico de estrategia el cual atiende lo siguiente: (a) la estrategia es un conjunto de metas, (b) las metas corporativas tratan de los resultados que las organizaciones pretenden lograr, (c) los logros que la organización pretende conseguir en el entorno en que se encuentra, (d) es importante visualizar lo que se desea tener, lo que se desea ser y (e) determinar una guía para que el administrador concrete a través de actividades los objetivos de la organización (Tilles, 1963). Los puntos antes descritos permiten tener mayor comprensión de la definición de estrategia. Como se conoce el administrador posee habilidades de pensador crítico y de pensador estratégico y es allí donde entra la teoría de juegos como parte de la estrategia de gestión.

Adicionalmente a lo expuesto en los párrafos anteriores, es preciso revisar el término administración estratégica o gestión estratégica, también llamada política o estrategia, identificada como dirección de empresas u organizaciones. Todos estos nombres han ido evolucionando de acuerdo a los requerimientos de la sociedad, la cual se exige que las organizaciones definan $\mathrm{y}$ tengan procesos bien adaptados y eficientes. La administración estratégica nutrida de tradición e historia que tomó como fuente de enseñanza de las escuelas de negocios incluye temas que son de interés primordial para la alta dirección, o para quien busca razones para el éxito y el fracaso de las organizaciones (Rumelt, Schendel, \& Teece, 1991). Esta persona que busca las razones de éxito no es otra que el "estratega debe crear, desplegar, combinar, gestionar y comunicar estos recursos sin la ayuda de los precios de mercado. (Lippman \& Rumelt, 2003), el administrador estratégico debe dominar los aspectos relacionados a su quehacer que son los siguientes: (a) los procesos de decisiones, (b) conocer los ambientes circunstanciales de la organización, (c) los problemas críticos de la organización y situaciones políticas, percibidas y evidenciadas a través de su estilo de liderazgo (Richardson, 1994) y que son parte del proceso administrativo y en este caso proceso administrativo estratégico.

De acuerdo a Hahn (2013) indicó que el proceso estratégico está compuesto por etapas que obligatoriamente deben cumplirse, para que la gestión alcance los mejores resultados planificados, estas fases son: (a) exploración del ambiente, que se refiere al análisis de las condiciones y las expectativas externas e internas hacia la sostenibilidad social corporativa, (b) formulación de estrategias, que hace referencia a la elaboración de misión, visón y establecimiento de objetivos a largo plazo y generación de estrategias, que permite tener una percepción del rol de empresa y su contribución a su entorno, de la misma forma permite conseguir y cómo trazar los objetivos de la organización y satisfacer las expectativas y alcanzar los objetivos, (c) implementación de estrategia, se refiere a los pasos o iniciativas que debe seguir la organización para el logro de sostenibilidad social corporativa y (d) evaluación de la estrategia, que hace referencia a valoración de los resultados, cómo medir y analizar el éxito; estos aspectos fueron propuestos por autores como David (2011), Wheelen y Hunger (2012).

\section{MATERIALES Y MÉTODOS}

Para la elaboración del presente trabajo se realizó una exhaustiva revisión de trabajos científicos conceptuales de varios autores que han realizado sus aportes y valoraciones acerca de la aplicación de la teoría de juegos en el ámbito administrativo y específicamente en la administración estratégica. Para ello se recopiló información científica en la base de datos de Thomsosn Reuters para garantizar que las fuentes utilizadas en este trabajo, sean confiables y tengan rigor científico y que las metodologías empleadas cumplan con las exigencias del mundo académico. Se realizó la búsqueda de la información a partir de las siguientes palabras claves Strategy, Strategic Management, Strategic Process, Game Theory, Relationship between game Theory y Strategic Management. Se empleó las habilidades de pensamiento crítico para poder desarrollar inferencias, reconocer asunciones, realizar 
deducciones, interpretaciones y evaluar argumentos de los autores consultados. Posterior a ello se estructuró una narración secuencial y lógica de los aportes encontrados y sus aplicaciones de manera que permita al lector una fácil comprensión de la temática tratada.

\section{ANÁLISIS Y DISCUSIÓN}

Si se aplica la teoría de juegos en el ámbito de las finanzas, se muestra que la teoría financiera es deficiente para proporcionar esclarecimiento a ciertos fenómenos observados en ese campo, lo que hace necesario la búsqueda de nuevas formas de trabajo, que conduzcan hacia metodologías cuantitativas que le permitan al profesional financiero a sacar inferencias a partir de los posibles resultados provistos a través del empleo de modelos matemáticos que brindan principalmente al campo de las finanzas corporativas algunas respuesta a los comportamientos inexplicables, propios de área del conocimiento empresarial. Esta aplicación de la teoría de juegos provee a esta disciplina precisión en lo referente a valoraciones de activos e interacción estratégica (Chatterjee \& Samuelson, 2014).

Otra aplicación de teoría de juegos es en la contabilidad porque ha proporcionado contestaciones concluyentes para brindar sentido práctico en esta área, aunque se han alcanzado importantes aportes en este ámbito aún falta mucho por hacer para que estos aportes se perciban como determinantes en el establecimiento de políticas. Uno de los aspectos que todavía tiene espacio para investigarse es el referente a las contradicciones en el diseño de contratos y medidas de rendimiento (Chatterjee \& Samuelson, 2014). "En teoría, la empresa debe ofrecer diferentes contratos a cada vendedor a pesar de que todos ellos están involucrados en esencialmente el mismo trabajo. Las realidades políticas y administrativas hacen esta recomendación difícil de implementar." (Chatterjee \& Samuelson, 2014). Este tipo de medidas se hacen difíciles de tomar cuando no se dispone de las herramientas administrativas que permitan aislar la subjetividad al realizar una decisión. Es en este aspecto donde la teoría de juegos ofrece al encargado de este tipo, departamentos de estrategias objetivas para que pueda realizar lo que la teoría contable por sí misma no puede lograr.

Según Chatterjee y Samuelson (2014) mencionaron que la teoría de juegos ha alcanzado una presencia importante en el área del
Marketing y la Comercialización, ya que en estas áreas de la administración se requiere la aplicación de estrategias que permitan objetivizar algunas de las situaciones propias de esta disciplina (Chatterjee \& Samuelson, 2014), por ejemplo tenemos que dotar de racionalidad a la relación con los proveedores y las formas en que se pretende ingresar en nuevos desafíos para ofrecer un producto o ingresar a un mercado a través de utilización de estrategias. Se debe mencionar que cada vez se le brinda un carácter más estructural a las áreas de comercialización de las empresas, a través de los modelos estructurales que son posibles mediante la aplicación de la teoría de juegos.

La Administración de Operaciones se la percibe como "un arte y como una ciencia; el arte de describir y modelar el problema, y la ciencia de resolver el modelo utilizando algoritmos matemáticos precisos" (Taha, 2012, p. 11). Según Willemain (1994) afirmó que "es una ciencia por las técnicas matemáticas que incorpora, y un arte porque el éxito de las fases que conducen a la solución del modelo matemático depende en gran medida de la creatividad y experiencia del equipo de Operaciones" (citado por Taha, 2012, p. 9). Por ello, en esta área tiene mucha acogida la teoría de juegos, ya que en esta parte de la materia administrativa se requiere las habilidades de pensador estratégico y de administrador estratégico, es decir "Esta teoría tiene que ver con situaciones de decisión en la que dos oponentes inteligentes con objetivos conflictivos compiten intensamente para superar al otro" (Taha, 2012, p. 541). La aplicación en esta área administrativa se hace evidente en temas como modelos de control de inventarios, en los que se tiene que decidir entre un proveedor $u$ otro, o se tiene que decidir entre una cantidad de compra para beneficiarse de las estrategias de ventas empleadas por los proveedores, o se tiene que decidir acerca del medio de transporte que se empleará para que los costos de operación no se incrementen. Otra evidente aplicación en el área de Operaciones es que en los juegos de suma, cero o constante implican conflicto de intereses, debido a que la selección de estrategias permiten neutralicen la intención del otro jugador para cambiar el juego (Taha, 2012). "Estas soluciones pueden ser en la forma de una sola estrategia o varias estrategias combinadas al azar" (Taha, 2012, p. 542).

Uno de los campos en los que se evidencia serias limitaciones de aplicación de la Teoría de juegos es en el campo de la Negociación 
debido a que la subjetividad humana juega un papel importante en esta área y justamente con la intervención de la teoría de juegos se desea objetivar los modelos de negociación existentes, porque coadyuva a la predicción de resultados de determinadas acciones a manera de receta, sin embargo el comportamiento de los involucrados ingresa a la función niveles de incertidumbre. No obstante, la teoría sugiere también las modificaciones que se pueden dar dependiendo de la estrategia empleada por uno de los jugadores y éste es el aspecto que muestra limitación de la aplicación ya que no ofrece garantía de predicción de los resultados (Camerer, 1991; Chatterjee \& Samuelson, 2014).

\section{CONCLUSIONES}

Cuando revisamos el aporte real que la teoría de juegos brinda a las ciencias administrativas se puede establecer como conclusión principal que la Teoría de Juegos tiene relación con la práctica administrativa debido a que brinda objetividad en cada uno de los procesos de la función administrativa, porque ofrece al administrador a través de la inferencia deducciones

\section{REFERENCIAS BIBLIOGRÁFICAS}

Andrews, K. (1971). Concept of corporate strategy. (st, nd and rd). Homewood, IL: Richard D Irwin.

Ansoff, I. (1991). Critique of Henry Mintzberg's "The design school: Reconsidering the basic premises of strategic management." Strategic Management Journal, 12(6), 449-461. http:// doi.org/10.1002/smj.4250120605.

Brandenburger, A., \& Nalebuff, B. (1995). The Right Game - Use Game-Theory to Shape Strategy. Harvard Business Review, 73(4), 57-71.

Camerer, C. (1991). Does Strategy Research Need Game Theory? Strategic Management Journal, $12,137-152$.

Chatterjee, K., \& Samuelson, W. (Eds.). (2014). Game theory and business applications (2. ed). Berlin: Springer.

David, F. (2011). Strategic management: concepts and cases (13th ed). Upper Saddle River, N.J: Prentice Hall.

Hahn, R. (2013). ISO 26000 and the Standardization of Strategic Management Processes for Sustainability and Corporate Social Responsibility. Business Strategy and the Environment, 22(7), 442-455. http://doi. org/10.1002/bse.1751.

Lippman, S., \& Rumelt, R. (2003). A Bargaining Perspective on Resource Advantage. Strategic Management Journal, 24(11), 1069-1086. de las posibilidades que tiene en función de la estructuración que le brinda la teoría de juegos, porque aísla la subjetividad en todos y cada uno de los procesos administrativos, le brinda al administrador condiciones propicias para que realice decisiones que lo lleven a obtener los mejores resultados para la organización. Sin embargo, a lo largo de la revisión de literatura realizada se ha podido percibir que la teoría de juegos tienen muchas aplicaciones y que podrían decirse que incluso en aquellas ciencias en las que parecería que es inaplicable tiene espacio. Debido a que al emplear bases matemáticas brinda escenarios estructurados de forma objetiva lo que hace en cada aplicación se pueda mostrar un argumento o fundamento lógico.

La abundante investigación realizada sobre los fundamentos técnicos y filosóficos de la teoría de juegos, nos hacen pensar que su vinculación con la gestión estratégica son fuertes (Rumelt et al., 1991), porque le provee de racionalidad a las interacciones propias de las ciencias sociales como la economía, comportamiento organizacional y en el aspecto práctico a los líderes de la organizaciones.

Richardson, B. (1994). Comprehensive Approach to Strategic Management: Leading across the Strategic Management Domain. Management Decision, 32(8), 27-41. http://doi.org/10.1108/ EUM0000000004314.

Rumelt, R., Schendel, D., \& Teece, D. (1991). Strategic Management and Economics. Strategic Management Journal, 12, 5-29.

Saloner, G. (1991). Modeling, game theory, and strategic management. Strategic Management Journal, 12(S2), 119-136. http:// doi.org/10.1002/smj.4250121009.

Taha, H. (2012). Investigación de operaciones. México D.F.: Pearson Educación.

Tarziján, J., \& Paredes, R. (2006). Organización industrial para la estrategia empresarial (2a ed.). México: Pearson Educación.

Tilles, S. (1963). How to Evaluate Corporate Strategy. Harvard Business Review, 41(4), 111-121.

Von Neumann, J., \& Morgenstern, O. (1944). Theory of games and economic behavior (First edition). Princeton, N.J. ; Woodstock: Princeton University Press.

Wheelen, T., \& Hunger, J. (2012). Strategic management and business policy: toward global sustainability (13th ed). Upper Saddle River, N.J: Pearson Prentice Hall. 